\title{
Magnetic Bead-Based Separation (MBS) of Pneumococcal Serotypes
}

\author{
Anna York ${ }^{1 *}$, Sidiya Mbodj ${ }^{1}$, Devyn Yolda-Carr ${ }^{1}$, Maikel Hislop ${ }^{1,2}$, Daniel M. Weinberger ${ }^{1}$, \\ Anne L. Wyllie ${ }^{1 *}$
}

${ }^{1}$ Department of Epidemiology of Microbial Diseases, Yale School of Public Health, New Haven, Connecticut, USA

${ }^{2}$ Department of Life Sciences and Chemistry, Utrecht University of Applied Sciences, Utrecht, Netherlands

*Corresponding Author

\begin{abstract}
There is often a need to separate a specific serotype of pneumococcus from a complex mixture of other pneumococcal strains. For instance, there could be a minority strain present at a low frequency in the nasopharynx that is difficult to identify and isolate by traditional culture. Or there might be a need to detect a minority serotype that has switched capsules in clinical samples or in laboratory experiments. We therefore developed a novel assay to separate mixed pneumococcal samples using serotype-specific antiserum and a magnetic bead-based separation method. We show using qPCR and colony counting methods, that six serotypes $(12 \mathrm{~F}, 23 \mathrm{~F}, 3,14,19 \mathrm{~A}$ and $15 \mathrm{~A})$ present as $\sim 0.1 \%$ of a dual serotype mixture can be enriched to between $10 \%$ and $90 \%$ of the final sample. This method is an important development for laboratory techniques which select for certain target serotype such as transformation and cocolonization experiments. With further development, this method has potential for clinical applications such as isolating pneumococcal strains from clinical samples.
\end{abstract}

\section{INTRODUCTION}

Streptococcus pneumoniae (pneumococcus) is an opportunistic pathogen that resides asymptomatically in the nasopharynx of many healthy adults and children worldwide. This asymptomatic colonization of the nasopharynx is a pre-requisite for the development of pneumococcal diseases such as upper respiratory tract infections (e.g., otitis media), lower respiratory tract infections (such as pneumonia), and invasive pneumococcal disease (IPD) (including meningitis and bacteraemia). Pneumococcal disease is a leading cause of morbidity and mortality in the young, elderly and the immunocompromised (1), with an estimated 1.6 million deaths occurring annually (2). The outermost layer of encapsulated strains is the capsular polysaccharide (CPS), and more than 100 antigenically different serotypes have been identified (3). Pneumococcal conjugate vaccines (PCV) are highly effective but only cover a small number of these serotypes. While pneumococcal disease declined following the introduction of PCVs, a concomitant increase in disease caused by non-vaccine serotypes occurred. This emergence of non-vaccine serotypes in carriage and invasive disease is called serotype replacement (4). Serotype replacement occurs for two reasons, first the opening of a new niche in which existing strains expressing capsules not targeted by the vaccine can thrive. Second, vaccine-targeted strains can acquire the capsule biosynthesis cassette from a different serotype, allowing them to evade vaccine-induced immunity. Serotype switching occurs when the cps locus from one $S$. pneumoniae serotype (or related species) is transferred into the genetic backbone of another $S$. pneumoniae serotype by transformation (5). Genetic exchange between two $S$. pneumoniae serotypes requires co-colonization of two or more serotypes.

In addition to naturally occurring serotype switches (6-8), researchers have been generating cps switch mutants in the lab for nearly 100 years. The first capsule switch experiments were 
accomplished by mixing avirulent, unencapsulated pneumococci with virulent, but killed, encapsulated strains, and injecting this mixture into a mouse. The capsule-switched strains could then be isolated from the mouse (9). More recently, generating cps switch mutants in the lab has been accomplished using various genetic cassettes $(10,11)$. These types of studies have permitted the generation of a number of capsule switch mutants, and this allows for detailed experimental evaluation of the relative importance of capsule and genetic background for different phenotypes $(8,12,13)$. All of the current approaches for generating capsule-switched variants require that the original capsule first be knocked out before the new capsule is introduced. Methods that allow direct switching of capsules between clinical strains would be more versatile and allow for higher throughput generation of capsule variants and more direct evaluation of mechanisms that influence capsule switching patterns. However, without a selectable marker, it has not previously been feasible to isolate rare capsule switch mutants from the parent strain.

We developed a magnetic bead-based separation (MBS) method which requires no selection 66 markers and can be used to extract live cells from a mixture of two pneumococci (of known serotypes) belonging to different serogroups.

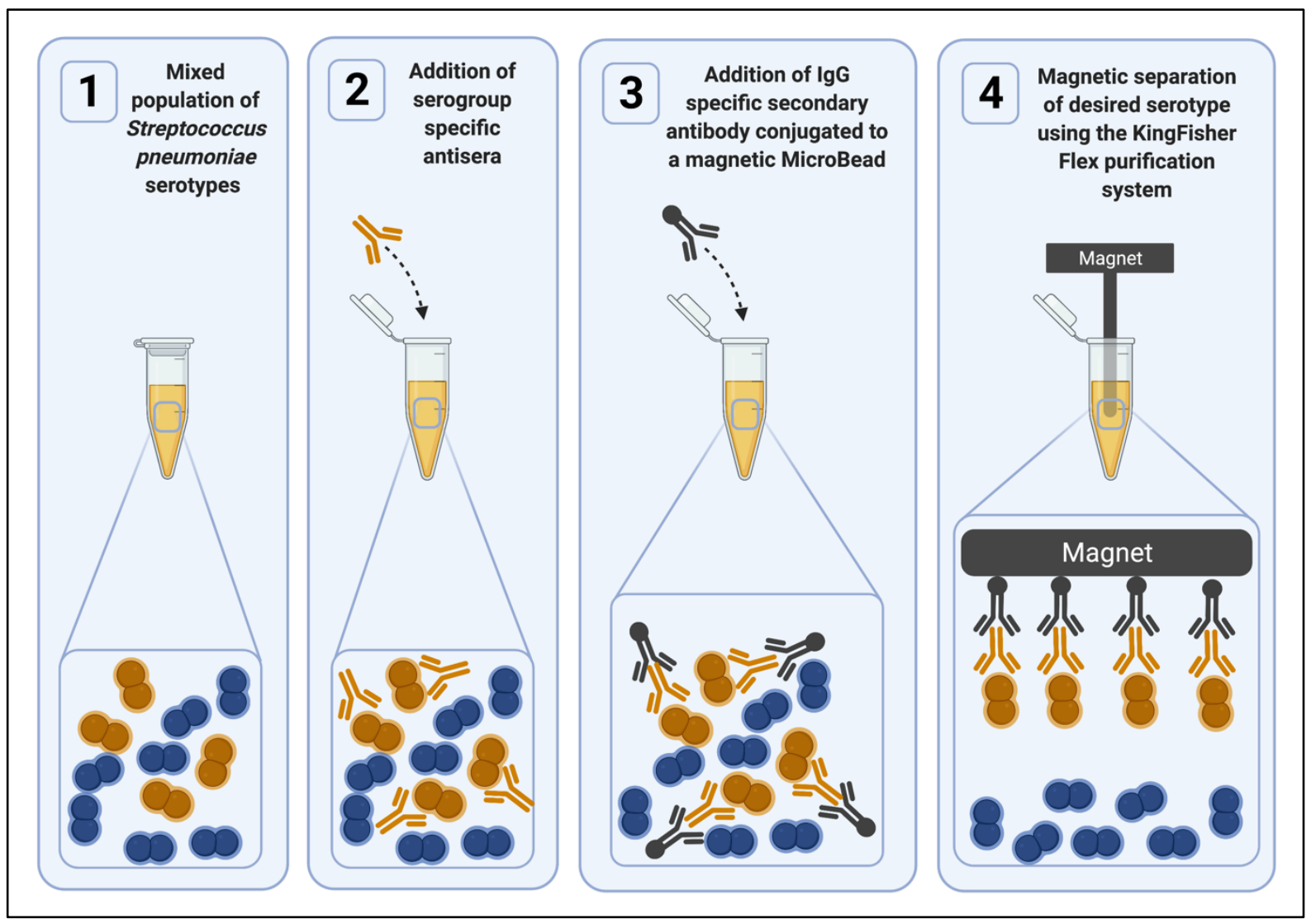

Figure 1- An overview of the Magnetic Bead-based Separation (MBS) method. To a dual serotype mixture (1), antisera specific for the desired serotype are added (2). Following brief wash steps, IgG-specific secondary antibody conjugated to a magnetic bead is incubated (3) and finally the desired cells are extracted using the KingFisher Flex Purification System (4). 


\section{METHODS}

Figure 1 summarizes the MBS method; briefly, a mixture of serotypes is incubated with antisera pool(s) unique to the desired serotype, then following wash steps is incubated with secondary antibody conjugated to a magnetic bead. The cells are extracted using the automated Kingfisher Flex Purification System and the eluate plated on blood agar plates.

\section{Magnetic Bead-Based Separation (MBS) Method}

Approximately $1 \times 10^{4}$ cells and $1 \times 10^{7}$ cells from two different serogroups of $S$. pneumoniae were mixed together ( $\sim 0.1 \%$ minority serotype). Cells were pelleted by centrifugation at 13,000 rpm and resuspended in $450 \mu$ Buffer 1 (1x Phosphate Buffered Saline with 1\% bovine serum albumin (BSA)). The resuspended sample was incubated at $4{ }^{\circ} \mathrm{C}$ on a shaking platform at 150 rpm for 1 hour. The two antisera pools specific for the minority serogroup were combined in a 1:1 ratio and diluted 50-fold in Buffer 1 . Next, $30 \mu \mathrm{l}$ of antisera mix was added to the sample and incubated at $4{ }^{\circ} \mathrm{C}$ on a shaking platform at $150 \mathrm{rpm}$ for 1 hour. The sample was centrifuged at 13,000 rpm for 5 mins, the supernatant was discarded, and the pellet was resuspended in 450 $\mu 1$ Buffer 1; this step was repeated again. Next, $20 \mu$ of Anti-Rabbit IgG MicroBeads (Miltenyi Biotech) were added, gently vortexed and incubated at $4{ }^{\circ} \mathrm{C}$ on a shaking platform at $150 \mathrm{rpm}$ for 30 minutes. The sample was extracted using the KingFisher ${ }^{\mathrm{TM}}$ Flex Purification System (ThermoFisher) with the protocol detailed in Figure S1. The eluted sample was resuspended by pipetting the sample in the elution well 50-100 times before transferring it to a new Eppendorf. Following transfer, the sample was thoroughly mixed by vortexing a minimum of 10 times for 5-10s with $5 \mathrm{sec}$ intervals.

To minimize cell losses, when supernatant was removed from cell pellets, $50 \mu 1$ of supernatant was always left on top of the pellet. The specific rabbit antiserum pools (SSI Diagnostica, Hillerød, Denmark) used for the MBS method, and the SSI ImmuLex ${ }^{\mathrm{TM}}$ Pneumotest Pools used for serotyping are outlined in Table S1.

\section{Proof of concept and primary analysis}

To demonstrate proof of concept for the MBS method we used three pairs of six different serotypes where one serotype in each pair was penicillin resistant and the other penicillin sensitive. It is important to note that different penicillin sensitivity is not necessary for separation but was instead used to make the quantification of the efficiency of this method easier. The three pairs were 12F and 23F (Pair 1), 3 and 14 (Pair 2) and 19A and 15A (Pair 3). Serotype 3 exists as two distinct morphologies; small non-mucoid colony variant (SCV) and mucoid variant (14). We therefore isolated SCV and mucoid variants and chose to work primarily with the SCV for three reasons; SCVs are easier to count, easier to isolate as single colonies (for serotyping) and less easy to distinguish from other serotypes based on morphology, thus reducing selection bias during the colony selection for serotyping. The MIC of each serotype was determined using penicillin E-strips, and then the exact concentration of penicillin for blood agar plates was determined experimentally by varying the penicillin concentration and plating out cells at known CFU/mL. The concentration of penicillin used in the blood agar plates was the concentration at which the resistant serotype grew equally well on a penicillin containing plate, as it did on a plain plate, whilst the susceptible serotype showed no growth on the penicillin containing plate but normal growth on a plain plate. For Pairs 1, 2 and 3 blood agar plates containing $0.018 \mu \mathrm{g} / \mathrm{mL}, 0.036 \mu \mathrm{g} / \mathrm{mL}$ and $0.18 \mu \mathrm{g} / \mathrm{mL}$ penicillin were used respectively. 
In all three pairs, Sample R is when the penicillin resistant serotype is the minority species, and Sample $\mathrm{S}$ is when the penicillin sensitive serotype is the minority species. Samples were plated out onto Tryptic Soy Agar (TSA) II + 5\% (v/v) Defibrinated Sheep Blood plates with and without penicillin, at two stages in the protocol; immediately prior to the first incubation (PRE), and after extraction (POST). In all cases $5 \mu$ of sample was serially diluted in $45 \mu \mathrm{PBS}$, in triplicate. For samples where the minority strain was penicillin resistant, $20 \mu \mathrm{l}$ of sample at a $10^{-1}$ dilution was plated on penicillin plates, while $20 \mu \mathrm{l}$ of sample at a $10^{-4}$ dilution was plated on plain blood agar plates. In samples where the majority serotype was penicillin resistant, 20 $\mu \mathrm{l}$ of sample at a $10^{-4}$ dilution was plated on both blood agar plates with and without penicillin. In addition to the diluted samples, $10 \mu 1$ of undiluted sample at the PRE and POST stage, and the remaining volume ( $\sim 40 \mathrm{ul})$ after elution was plated on plain blood agar plates, to provide DNA for qPCR experiments conducted to establish separation efficiency. In all cases $10 \mu 1$ or $20 \mu \mathrm{l}$ samples were pipetted onto the blood agar plate and the plate was then tilted to allow the sample to run down the length of the plate. The agar plates were incubated overnight at $37^{\circ} \mathrm{C}$ and $5 \% \mathrm{CO}_{2}$.

\section{Secondary analyses}

To establish if separation efficiency was similar for both mucoid (Muc) and single colony variants (SCV) of Serotype 3, two additional pairs; 23F and 3SCV (Pair 4), and 23F and 3Muc (Pair 5) were investigated. These experiments were conducted in duplicate, and efficiency assessed by colony counting and qPCR methods. Pair 4 and 5 used blood agar plates containing $0.072 \mu \mathrm{g} / \mathrm{mL}$ penicillin.

To investigate the effect of initial proportion of minority serotype on the efficiency of separation, 23F and 12F (Pair 1) were again used. The initial amount of majority serotype (12F) was kept constant at $1 \times 10^{7} \mathrm{CFUs}$, while the minority serotype $(23 \mathrm{~F})$ was varied $\left(5 \times 10^{4}, 1 \times 10^{4}\right.$, $5 \times 10^{3}$ and $\left.1 \times 10^{3}\right)$. These experiments were conducted once for each dilution, and efficiency was assessed by colony counting and qPCR methods.

The experiments above were conducted using 2 pooled antisera that were specific for the minority serotype. We investigated whether a single pool of antisera could also be used. This is important because certain pairs of serotypes can only be distinguished by one pool. Serotype pairs which could not be distinguished based on penicillin sensitivity (and therefore could not be assessed by colony counting methods), were used for this analysis, and for pairs which shared a common antisera pool, only the unique antisera was used. These experiments were conducted once for each condition, and efficiency was assessed by qPCR alone.

\section{Colony counting to quantify separation efficiency}

Colonies were counted and the mean colony number was determined, which was then used for downstream analysis. The following equations for Sample $\mathrm{R}$ and Sample $\mathrm{S}$ were used to determine the percentage of the minority serotype present at each time point.

\section{Sample R Equation}

$$
\% \text { minority }=\left(\frac{\# \text { colonies }(\text { pen plate })}{\# \text { colonies }(\text { plain plate })}\right) \times 100
$$

\section{Sample S Equation}




$$
\% \text { minority }=\left(\frac{\# \text { colonies }(\text { plain plate })-\# \text { colonies }(\text { pen plate })}{\# \text { colonies }(\text { plain plate })}\right) \times 100
$$

\section{Serotyping of colonies to confirm separation efficiency}

176 Eight colonies were picked at random from the plain blood agar elution plates and expanded to create a lawn on $1 / 8^{\text {th }}$ of a plain blood agar plate, and grown overnight at $37{ }^{\circ} \mathrm{C}$ with $5 \% \mathrm{CO}_{2}$. The serotype of each lawn was then confirmed by testing each of the four antisera pools specific to both the majority and the minority serotype in the pair, using ImmuLex ${ }^{\mathrm{TM}}$ Pneumotest (SSI

180 Diagnostica) reagents.

\section{Real-Time qPCR to confirm separation efficiency}

183 Colonies/lawns from each sample, grown on plain blood agar plates, were harvested into 200 $\mu \mathrm{l}$ PBS using a cotton swab and the DNA was extracted using a DNeasy Blood and Tissue Kit (QIAGEN) as per the manufacturers protocol. DNA concentration was measured using Qubit $^{\mathrm{TM}}$ as per the manufacturers protocol. A no-template negative control was included for each primer pair, and a standard curve (positive control) was constructed using genomic DNA from each of the six serotypes under investigation. The serotype specific primers used were described previously (15). Each qPCR reaction was $25 \mu$ l total volume, consisting of $\mathrm{iQ}^{\mathrm{TM}}$ SYBR ${ }^{\circledR}$ Green Supermix (BioRad), $5 \mathrm{ul}$ of template DNA and $20 \mathrm{nM}$ of each primer. The real time qPCR was run on a BioRad CFX96 ${ }^{\mathrm{TM}}$ Real-Time qPCR System. The cycling conditions were 1 cycle of denaturation at $95{ }^{\circ} \mathrm{C}$ for 10 minutes, followed by 40 cycles of $95{ }^{\circ} \mathrm{C}$ for 15 seconds and $60{ }^{\circ} \mathrm{C}$ for 1 minute for amplification, and a melt curve from $65{ }^{\circ} \mathrm{C}$ to $95{ }^{\circ} \mathrm{C}$ in increments of $0.5^{\circ} \mathrm{C}$. For each sample, amplification with primer pairs from both the minority and majority serotype was conducted in duplicate, the mean of duplicates was used for downstream analysis. The concentration of each serotype in a sample was determined by comparing the $\mathrm{Ct}$ value to the standard curve for the corresponding serotype. 


\section{RESULTS}

For all six serotypes, the minority serotype was successfully enriched from $\sim 0.1 \%$ starting percentage to between $13 \%$ (serotype 14) and 90\% (serotype 3 ) post MBS, corresponding to a 100-to-900-fold enrichment (Figure 2a). The final percentage of the minority varied between serotypes but was relatively consistent between the three replicates. There was generally good concordance in the estimated MBS efficiency as determined by the qPCR and colony counting (Figure 2b), however efficiency determined by colony counting seemed to be higher and lower than with qPCR for serotype 14 and 3, respectively. Eight colonies from each elution plate were selected at random and in every single case, minority serotype colonies were identified by serotyping (Table 1). This demonstrates that this technique can be used to recover a desired serotype from a dual mixture.

212

a)

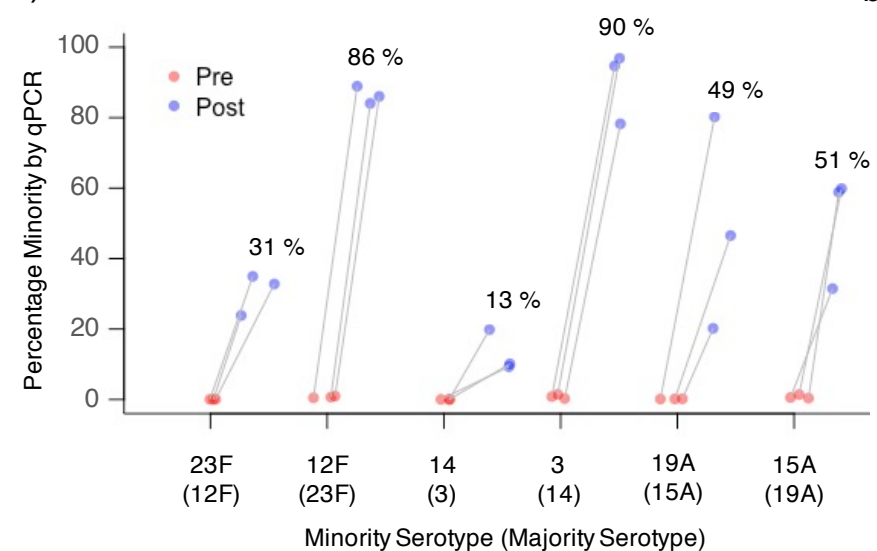

b)

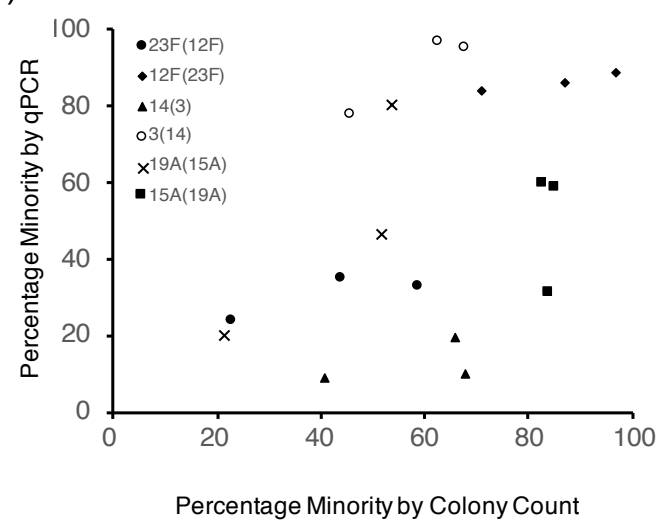

Figure 2- Efficiency of minority enrichment using the MBS method on six serotypes. a) Percentage minority serotype present prior to MBS (Pre) and after MBS (Post). The average percentage minority in the post sample is presented above the data points, b) Percentage minority serotype present after MBS (Post) as determined by qPCR and colony counting. Minority and majority serotypes are displayed in the legend in the following format, minority(majority). Results are shown in triplicate for each of three serotype pairs where each serotype of the pair was tested as the minority serotype.

A secondary analysis was conducted to identify whether serotype 3Muc was also enriched with a similar efficiency as serotype $3 \mathrm{SCV}$, and to gain insight into how separation efficiency varies when the majority serotype of the pair is altered. MBS was conducted on Pair 4 (23F and 3SCV and Pair 5 (23F and 3Muc). The percentage enrichment for both 23F and 3 remained similar even when the majority serotype of the pair was altered (Figure 3). Furthermore, it demonstrates that this MBS method permits successful enrichment of both SCV and mucoid variants of serotype 3, and that the efficiency is similar regardless of the morphology. In all cases minority serotype single colonies were isolated from the elution plate by selection of single colonies and confirmed to be the desired serotype using SSI latex agglutination (Table 1). 
a)

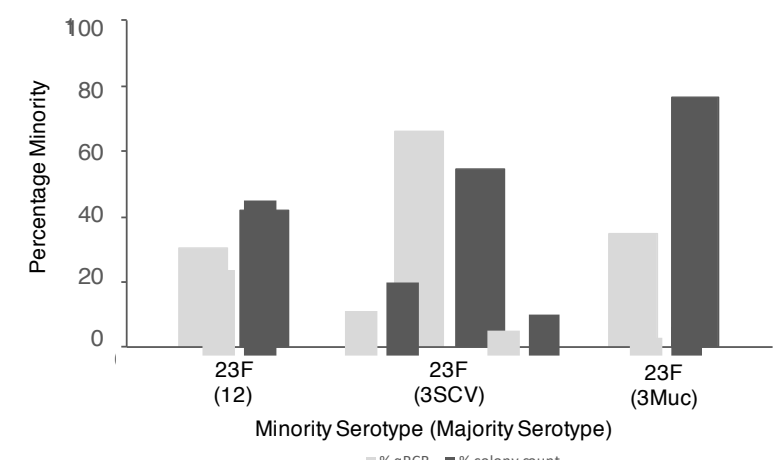

b)

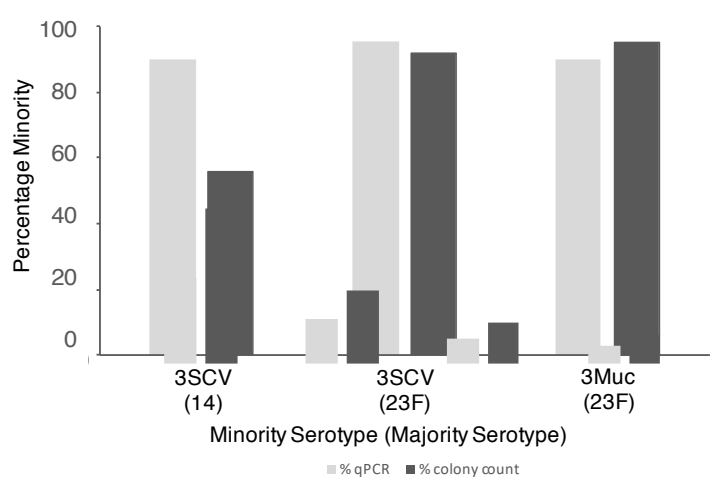

Figure 3 - Final percentage of minority serotype after MBS. Averages of triplicate results from Figure 1 are shown for $23 F$ (when with a majority of $12 F$ ) and 3 (when with a majority of 14). Averages of duplicate results were plotted for $23 F$ (when with a majority of $3 \mathrm{SCV}$ ), $23 \mathrm{~F}$ (when with a majority of $3 \mathrm{Muc}$ ), $3 \mathrm{SCV}$ (when with a majority of $23 \mathrm{~F}$ ) and $3 \mathrm{Muc}$ (when with a majority of 23F). Percentage minority from both colony counting and qPCR methods is shown.

Table 1 - Total number of colonies on the plain blood agar elution plate (out of eight selected at random) that were positive for the minority serotype by SSI latex agglutination. Positive results reported were those that tested positive with minority setotype antisera and negative with majority serotype antisera.

\begin{tabular}{|c|c|c|c|c|}
\hline \multicolumn{2}{|c|}{ Serotype Pairs } & \multicolumn{3}{|c|}{$\begin{array}{c}\text { Number of colonies of the minority serotype following } \\
\text { MBS } \\
\text { (from eight selected at random from plain blood agar } \\
\text { plates) }\end{array}$} \\
\hline $\begin{array}{l}\text { Minority } \\
\text { Serotype }\end{array}$ & \begin{tabular}{|l|} 
Majority \\
Serotype
\end{tabular} & Rep1 & Rep2 & Rep3 \\
\hline $23 \mathrm{~F}$ & $12 \mathrm{~F}$ & 5 & 5 & 5 \\
\hline $12 \mathrm{~F}$ & $23 \mathrm{~F}$ & 8 & 6 & 5 \\
\hline 14 & $3(\mathrm{SCV})$ & 4 & 4 & 3 \\
\hline $3(\mathrm{SCV})$ & 14 & 7 & 6 & 6 \\
\hline $19 \mathrm{~A}$ & $15 \mathrm{~A}$ & 1 & 7 & 3 \\
\hline $15 \mathrm{~A}$ & $19 \mathrm{~A}$ & 7 & 6 & 5 \\
\hline $23 \mathrm{~F}$ & $3(\mathrm{SCV})$ & 4 & 2 & $\mathrm{~N} / \mathrm{A}$ \\
\hline $3(\mathrm{SCV})$ & $23 \mathrm{~F}$ & 6 & 8 & $\mathrm{~N} / \mathrm{A}$ \\
\hline $23 \mathrm{~F}$ & 3(Muc) & 6 & 8 & $\mathrm{~N} / \mathrm{A}$ \\
\hline 3(Muc) & $23 \mathrm{~F}$ & 5 & 7 & N/A \\
\hline
\end{tabular}

The primary analysis specifically used serotype pairs that could be distinguished using two unique pools of antisera. MBS was then tested on eight serotype pairs using only a single antisera pool. A total of six antisera pools (H, P, B, E, R, H and Q) were tested and all were able to successfully enrich a $\sim 0.1 \%$ minority serotype to between $10 \%$ and $99 \%$ in the final sample (Figure 4a). 
a)

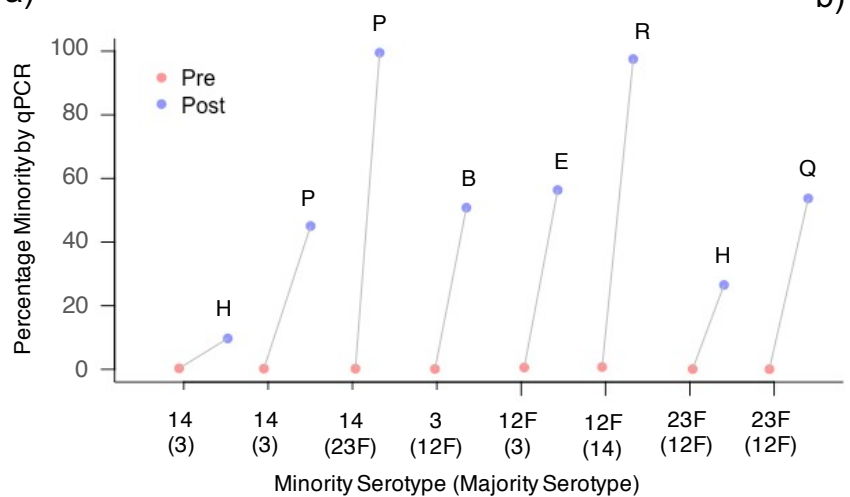

b)

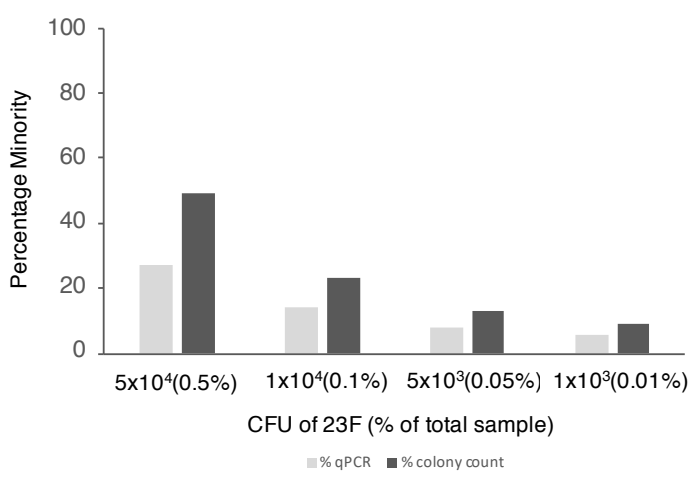

Figure 4 - Efficiency of minority enrichment using the MBS method on eight serotypes. a) Percentage minority serotype present prior to MBS (Pre) and after MBS (Post), b) Percentage minority $23 F$ present after MBS (Post) as determined by $q P C R$ and colony counting. Minority and majority serotypes are displayed in the following format, minority (majority).

Results shown are singlicate data points only.

252

253

254

255

256

257

258

259

260

261

262

263

264

265

266

267

268

269

270

271

272

273

274

275

276

277

278

279

280

281

282

283

284

285

Additional analysis aimed to determine whether enrichment was constant at different \% minorities. The $23 \mathrm{~F}$ and $12 \mathrm{~F}$ pair were used with the majority serotype $(12 \mathrm{~F})$ remaining constant at $1 \times 10^{7} \mathrm{CFUs}$ and the minority serotype (23F) at four different concentrations in the initial sample. Enrichment of the minority serotype can be achieved even when the starting percentage of a minority serotype is as low as $1 \times 10^{3} \mathrm{CFUs}$. However, as the initial \% minority decreases the percentage minority recovered following MBS also decreases. For initial samples containing $5 \times 10^{4}, 1 \times 10^{4}, 5 \times 10^{3}$ and $1 \times 10^{3}$ CFU's of minority serotype $23 \mathrm{~F}$, the corresponding percentage of $23 \mathrm{~F}$ present in the final samples were $27 \%, 14 \%, 8 \%$ and $6 \%$ respectively as determined by qPCR, or $49 \%, 23 \%, 13 \%$ and $9 \%$ respectively as determined by colony counting (Figure 4b).

\section{DISCUSSION}

We developed this MBS method to enrich for a desired serotype from a mixed-serotype sample in a laboratory setting. We demonstrate using six minority serotypes $(23 \mathrm{~F}, 12 \mathrm{~F}, 3,14,15 \mathrm{~A}$ and $19 \mathrm{~A}$ ) that a minority serotype can be enriched from $\sim 0.1 \%$ of an initial mixed serotype sample to up to $90 \%$ in the final sample. This method permits separation of a mixture of pneumococcal serotypes in just a few hours. We demonstrate that enrichment of a serotype can occur even when a serotype is present at only $0.01 \%$ of the total sample $\left(1 \times 10^{3}\right.$ minority serotype with $1 \times 10^{7}$ majority serotype). We also show that it is possible to achieve good separation with only one unique antisera, meaning that serotypes with cross-reactivity to one antisera can still be separated using this method. In addition, we show that MBS can be used to enrich for serotypes will more unique capsules; two serotype 3 variants (single colony and mucoid) and serotype 14 (which has an uncharged capsule).

This technique has a number of potential applications, including allowing for the quick and easy purification of cps transformants from a mix of non-transformed cells (the recipient serotype). This method eliminates the need for selection markers (10) and is suitable for higher throughput mutant generation. The method may also be useful for co-carriage studies or competition assays where separating the serotypes for downstream analysis may beneficial. In addition, this technique may be used in field epidemiological research to isolate minority serotypes in samples obtained from multiply colonized individuals. Previous research shows that $52 \%$ of Dutch primary school children tested positive for multiple pneumococcal serotypes (16), however, conventional serotyping methods often result in an underestimation of multiply 
colonized individuals (17). Detection of multiple serotypes is possible using serologic, biochemical (Mass Spectroscopy and nuclear Magnetic Resonance), and genotypic (sequencing, qPCR and microarrays) methods, however, until now, attempting to isolate minority serotypes by conventional methods (single colony selection) has been laborious and time consuming (18).

In the primary analysis we show that all six of the minority serotypes investigated can be successfully separated from a majority serotype with the percentage minority present post MBS ranging from $13 \%$ for serotype 14 , to $90 \%$ for serotype 3 . Two methods; colony counting, and qPCR were employed in order to assess efficiency of the MBS method. The estimates from both methods were broadly concurrent but there are a few examples where the efficiency estimates do differ. This may be explained by the formation of varying chain lengths in pneumococcus, such that if the two serotypes in a pair form vastly different length chains, the estimations of efficiency may be biased. A serotype that readily forms chains would result in an underestimation of its presence in the sample using the colony counting method, but qPCR would provide a more accurate estimation. Despite some differences in efficiency estimates between colony counting and qPCR methods, we were able to successfully isolate minority serotype colonies post MBS in all cases. This demonstrates a tangible utility for this method in the laboratory setting. When separating a mixture of cells only a small number of colonies must be isolated in order to identify the desired serotype. This method therefore allows for the easy recovery of serotype specific $S$. pnuemoniae colonies.

In the secondary supporting analysis, minority serotype $23 \mathrm{~F}$ was paired with a majority serotype of either $12 \mathrm{~F}$ or 3 , and minority serotype 3 was paired with a majority serotype of either 14 or 23F. With minority 23F, some variation in efficiency of MBS was noted when the majority serotype was changed, however for minority serotype 3, the enrichment efficiency remained very similar despite the change in majority serotype pair. This suggests that the serotype with which the minority is mixed may have some impact on the efficiency of MBS, but it is likely primarily determined by the avidity of the antisera for the desired serotype. Unlike the majority of pneumococcal serotypes, serotype 3 utilizes the synthase-dependent pathway for CPS production, resulting in non-covalently bound CPS which can be released from the glycolipids or synthase (18). Since the release of serotype 3 CPS reduces the protective effect of anti-Type 3 CPS antibodies (19), we were surprised to find that the MBS method can successfully extract serotype 3 from a mixed sample. This success may be explained by the fact that the cells are not actively growing and likely therefore not releasing CPS into the environment. Furthermore, it is intriguing but reassuring that the efficiency of enrichment between mucoid and SCV serotype 3 is very similar; this MBS method can therefore be successfully used on serotype 3 samples, which are of particular interest due to the reduced effectiveness of PCV13 on serotype 3 IPD (20-22).

Demonstrating that successful separation can also be achieved using only a single antisera pool makes this method more versatile and accessible by increasing the number of possible serotype combinations that can be separated. Using only one antisera pool will also decrease the cost. As expected, we demonstrate that the efficiency of enrichment achieved by each of the two antisera pools is not equal and therefore, depending on the desired serotype one antisera may be preferred over another. MBS of serotype 14 from a majority serotype 3, using both antisera Pool $\mathrm{H}$ and Pool P, resulted in the final sample containing $\sim 13 \%$ of serotype 14 . However, use of only Pool $\mathrm{H}$ or Pool P, at an equal final volume to the combined pools, resulted in serotype 14 being $10 \%$ and $45 \%$ of the final samples respectively. Therefore, in this example, Pool $\mathrm{P}$ alone achieves the greatest efficiency of MBS, but in the absence of knowing which antisera is 
more efficient, and if the serotype pairs permit dual use, it would be prudent to combine both antisera pools. Furthermore, we confirm that the overall efficiency of enrichment achieved by any antisera pool, is not only dependent upon the minority serotype alone, but also the majority serotype. The final percentage of serotype 14 following MBS (using Pool P) from a majority serotype $23 \mathrm{~F}$, is $99 \%$, more than double the percentage of serotype 14 present following MBS (using Pool P) from a majority serotype 3.

A key limitation of this method is that due to cross-reactivity within serogroups, this technique can only be used to separate $S$. pneumoniae serotypes belonging to different serogroups. Another limitation is the total proportion of minority cells that can be recovered. While enrichment from $0.1 \%$ up to $>10 \%$ has been demonstrated, it is worth noting that only a small proportion $(\sim 1 \%)$ of the total minority cells present in the initial mixture are successfully extracted. This may be overcome increasing antibody incubation periods or antibody concentration to increase binding capacity. Alternatively, use of a type-specific antisera or a monoclonal antibody instead of pooled antisera may improve the overall recovery, however this would simultaneously increase the cost of this technique. Despite these limitations, the MBS method remains less labor intensive and less expensive than the more traditional methods available, it also opens up doors to experiments that would previously have not been possible.

In conclusion, MBS allows for the successful enrichment of a minority serotype from a dual sample containing two $S$. pneumoniae serotypes belonging to different serogroups. Using this method, an initial sample containing $0.01-0.1 \%$ of a desired serotype, can be enriched to up to $90 \%$ in the final sample. Enrichment to between 10 and $90 \%$ was demonstrated for six minority serotypes, and half of the commercially available antisera pools (Pools B, E, H, P, Q, R and $S)$ were tested. This MBS method can be used for the separation of mixed serotype cultures of S. pneumoniae and will serve as an important technique in the generation of capsule switch mutants. It may also be utilized in co-colonization studies, and for isolation of minority serotypes from contaminated or mixed cultures. With further development, it may be possible to use this technique to isolate serotype specific $S$. pneumoniae from more complex mixtures, such as samples containing increased numbers of serotypes, or clinical samples such as saliva.

\section{AUTHOR CONTRIBUTIONS}

DMW and ALW conceptualized the study; AY, DMW and ALW designed the experiments; AY, SM, MH and DYC conducted the experimental procedures; AY and DW conducted data analysis; AY wrote the manuscript; DMW, ALW, SM, MH and DYC reviewed the manuscript; ALW and DMW supervised the work.

\section{AUTHOR DECLARATION}

DMW has received consulting fees from Pfizer, Merck, GSK, and Affinivax and is PI on research grants from Pfizer and Merck to Yale. ALW has received consulting fees from Pfizer and is PI on research grants from Pfizer to Yale. The other authors declare no conflict of interest.

\section{FUNDING}

This work is supported by R01AI123208 from NIAID/NIH (DMW). The content is solely the responsibility of the authors and does not necessarily represent the official views of the National Institutes of Health. 
Table S1 - Serotypes and corresponding antisera pools used for MBS (rabbit antiserum; SSI

\begin{tabular}{|l|l|l|}
\hline Serotype & $\begin{array}{l}\text { Pooled Antisera } \\
\text { for Neufeld }\end{array}$ & $\begin{array}{l}\text { ImmuLex } \\
\text { Pneumococcus } \\
\text { Antisera }\end{array}$ \\
\hline $12 \mathrm{~F}$ & $\begin{array}{l}\text { Pool E \#16733 } \\
\text { Pool R \#16741 }\end{array}$ & $\begin{array}{l}\text { Pool E \#52394 } \\
\text { Pool R \#52401 }\end{array}$ \\
\hline $23 \mathrm{~F}$ & $\begin{array}{l}\text { Pool H \#16736 } \\
\text { Pool Q \#16740 }\end{array}$ & $\begin{array}{l}\text { Pool H \#52397 } \\
\text { Pool Q \#52400 }\end{array}$ \\
\hline 3 & $\begin{array}{l}\text { Pool B \#16728 } \\
\text { Pool R \#16741 }\end{array}$ & $\begin{array}{l}\text { Pool B \#52391 } \\
\text { Pool R \#52401 }\end{array}$ \\
\hline 14 & $\begin{array}{l}\text { Pool P \#16739 } \\
\text { Pool H \#16736 }\end{array}$ & $\begin{array}{l}\text { Pool P \#52399 } \\
\text { Pool H \#52397 }\end{array}$ \\
\hline $19 \mathrm{~A}$ & $\begin{array}{l}\text { Pool B \#16728 } \\
\text { Pool P \#16739 }\end{array}$ & $\begin{array}{l}\text { Pool B \#52391 } \\
\text { Pool P \#52399 }\end{array}$ \\
\hline $15 \mathrm{~A}$ & Pool H \#16736 \\
Pool S \#16742 & $\begin{array}{l}\text { Pool H \#52397 } \\
\text { Pool S \#52402 }\end{array}$ \\
\hline
\end{tabular}

Figure S1 - KingFisher Flex MBS Protocol

Pick-Up

CollectBeads 1

Mix 1

Beginning of step

Mixing / heating:

End of step

ReleaseBeads1

Leave samples

Collect count

Collect time [s]

wash 1

Precollect

Release beads

Mixing time, speed

Heating during mixing

Postmix

Collect count

Collect time [s]

elution

Release time, speed

00:00:05, Fast

leave plate

\section{2}

5

\section{Supplemental File - Raw Data}




\section{References}

1. Prevention of pneumococcal disease : recommendations of the Advisory Committee on Immunization Practices (ACIP) [Internet]. [cited 2021 Apr 23]. Available from: https://stacks.cdc.gov/view/cdc/5708

2. WHO | Pneumococcal disease [Internet]. WHO. World Health Organization; [cited 2021 Apr 23]. Available from: https://www.who.int/ith/diseases/pneumococcal/en/

3. Ganaie F, Saad JS, McGee L, van Tonder AJ, Bentley SD, Lo SW, et al. A New Pneumococcal Capsule Type, 10D, is the 100th Serotype and Has a Large cps Fragment from an Oral Streptococcus. McDaniel LS, editor. mBio. 2020 May 19;11(3):e00937-20, /mbio/11/3/mBio.00937-20.atom.

4. Weinberger DM, Malley R, Lipsitch M. Serotype replacement in disease after pneumococcal vaccination. The Lancet. 2011 Dec 3;378(9807):1962-73.

5. Nahm MH, Brissac T, Kilian M, Vlach J, Orihuela CJ, Saad JS, et al. Pneumococci Can Become Virulent by Acquiring a New Capsule From Oral Streptococci. J Infect Dis. 2019 Sep 7;jiz456.

6. Chochua S, Metcalf BJ, Li Z, Walker H, Tran T, McGee L, et al. Invasive Serotype 35B Pneumococci Including an Expanding Serotype Switch Lineage, United States, 20152016. Emerg Infect Dis. 2017 Jun;23(6):922-30.

7. Moore MR, Gertz J Robert E, Woodbury RL, Barkocy-Gallagher GA, Schaffner W, Lexau C, et al. Population Snapshot of Emergent Streptococcus pneumoniae Serotype 19A in the United States, 2005. J Infect Dis. 2008 Apr 1;197(7):1016-27.

8. Croucher NJ, Kagedan L, Thompson CM, Parkhill J, Bentley SD, Finkelstein JA, et al. Selective and Genetic Constraints on Pneumococcal Serotype Switching. Hughes D, editor. PLOS Genet. 2015 Mar 31;11(3):e1005095.

9. Griffith Fred. The Significance of Pneumococcal Types. J Hyg (Lond). 1928 Jan;27(2):113-59.

10. Li Y, Thompson CM, Lipsitch M. A Modified Janus Cassette (Sweet Janus) to Improve Allelic Replacement Efficiency by High-Stringency Negative Selection in Streptococcus pneumoniae. Beall B, editor. PLoS ONE. 2014 Jun 24;9(6):e100510.

11. Echlin H, Rosch JW. Advancing Genetic Tools in Streptococcus pneumoniae. Genes. 2020 Sep;11(9):965.

12. Kelly T, Dillard JP, Yother J. Effect of genetic switching of capsular type on virulence of Streptococcus pneumoniae. Infect Immun. 1994 May 1;62(5):1813-9.

13. Hathaway LJ, Brugger SD, Morand B, Bangert M, Rotzetter JU, Hauser C, et al. Capsule Type of Streptococcus pneumoniae Determines Growth Phenotype. Orihuela CJ, editor. PLoS Pathog. 2012 Mar 8;8(3):e1002574.

14. Allegrucci M, Sauer K. Characterization of Colony Morphology Variants Isolated from Streptococcus pneumoniae Biofilms. J Bacteriol. 2007 Mar 1;189(5):2030-8. 
15. Pimenta FC, Roundtree A, Soysal A, Bakir M, McGee L. Sequential Triplex Real-Time PCR Assay for Detecting 21 Pneumococcal Capsular Serotypes That Account for a High Global Disease Burden. J Clin Microbiol. 2013;51(2):6.

16. Wyllie AL, Chu MLJN, Schellens MHB, Gastelaars J van E, Jansen MD, Ende A van der, et al. Streptococcus pneumoniae in Saliva of Dutch Primary School Children. PLOS ONE. 2014 Jul 11;9(7):e102045.

17. Huebner RE, Dagan R, Porath N, Wasas AD, T M, Klugman KP. LACK OF UTILITY OF SEROTYPING MULTIPLE COLONIES FOR DETECTION OF SIMULTANEOUS NASOPHARYNGEAL CARRIAGE OF DIFFERENT PNEUMOCOCCAL SEROTYPES. Pediatr Infect Dis J. 2000 Oct;19(10):1017-20.

18. Geno KA, Gilbert GL, Song JY, Skovsted IC, Klugman KP, Jones C, et al. Pneumococcal Capsules and Their Types: Past, Present, and Future. Clin Microbiol Rev. 2015 Jul;28(3):871-99.

19. Choi EH, Zhang F, Lu Y-J, Malley R. Capsular Polysaccharide (CPS) Release by Serotype 3 Pneumococcal Strains Reduces the Protective Effect of Anti-Type 3 CPS Antibodies. Burns DL, editor. Clin Vaccine Immunol. 2016 Feb;23(2):162-7.

20. Horácio AN, Silva-Costa C, Lopes JP, Ramirez M, Melo-Cristino J, Infections PG for the $\mathrm{S}$ of $\mathrm{S}$, et al. Serotype 3 Remains the Leading Cause of Invasive Pneumococcal Disease in Adults in Portugal (2012-2014) Despite Continued Reductions in Other 13-Valent Conjugate Vaccine Serotypes. Front Microbiol [Internet]. 2016 [cited 2021 Apr 25];7. Available from: https://www.frontiersin.org/articles/10.3389/fmicb.2016.01616/full

21. Silva-Costa C, Brito MJ, Pinho MD, Friães A, Aguiar SI, Ramirez M, et al. Pediatric Complicated Pneumonia Caused by Streptococcus pneumoniae Serotype 3 in 13-Valent Pneumococcal Conjugate Vaccinees, Portugal, 2010-2015. Emerg Infect Dis. 2018 Jul;24(7):1307-14.

22. Slotved H-C, Dalby T, Harboe ZB, Valentiner-Branth P, Casadevante VF de, Espenhain $\mathrm{L}$, et al. The incidence of invasive pneumococcal serotype 3 disease in the Danish population is not reduced by PCV-13 vaccination. Heliyon. 2016 Nov 1;2(11):e00198. 